\title{
Beiträge zur Koehlerschen Krankheit des Kahnbeins am Fuße bei Kindern.
}

Von Privatdozent Dr. Erich Sonntag, Oberarzt an der Poliklinik der chirurgischen I'niversiätsklinik zu Leipzig.

(Mit 7 Abbildungen.)

Alban Koehler hat im Jahre igo8 auf der 4. Tagung der Deutschen Röntgengesellschaft zum ersten Male berichtet über einc häufige, bisher anscheinend unbekannte Erkrankung einzelner kindlicher Knochen, insonderheit über eine eigentümliche Affektion des Os naviculare pedis. Seitdem sind eine Reihe ähnlicher Fälle unter der Bezeichnung der ,Koehlerschen Krankheit" mitgeteilt worden; Koehler konnte in der Literatur im Jahre I9I4 26 Fälle und ich jetzt 48 Fälle auffinden (den Fall Dobisch rechne ich - wie andere Autoren - nicht dazu, da er offenbar nicht hierher gehört). Ich selbst sah unter den Fällen der hiesigen Poliklinik, welche ich seit $1 / 2$ Jahr führe, 2 sehr typische Fälle; 2 andere Fälle, welche früher hier beobachtet wurden, hat Bachmann im Jahre 19I4 auf der 10. Tagung der Deutschen Röntgengesellschaft in der Diskussion zu Koehlers 2. Vortrag mitgeteilt. Da die Zahl der veröffentlichten Fälle noch eine recht bescheidene ist (etwa ein halbes Hundert) und da noch keine genügende Klarheit über das Krankheitsbild, insonderheit über dessen Wesen und Ursache besteht, so erscheinen die von mir beobachteten Fälle der Mitteilung wert.

F a 11 I. K. S., 6 j. Knabe.

Vorgeschichte: Eltern und 4 Geschwister gesund. 5. Kind. Mutter hatte zum SchluB noch eine Fehlgeburt. Keine fruheren Krankheiten, insonderheit keine Rachitis, Tuberkulose oder Lues; Zahnung und Laufenlernen zur rechten Zeit. Seit 2 Tagen, im Anschluß an einen längeren Spaziergang, aber ohne jegliches Trauma, bestehen Schmerzen am r. FuB innenseits; auch hinkte der Knabe leicht und trat vorwiegend mit dem äußeren Fußrand auf; am näch- 
sten Tage konnte er nicht mehr zur Schule gehen; da die Schmerzen bei Schonung und feuchten Umschlägen nicht nachließen, bringen ihn die Eltern in die hiesige Poliklinik.

A uf nahmebefund: Allgemeinzustand o. B., insbesondere ohne Zeichen von Körperschwäche, Myxödem, Blutarmut, Rachitis, Tuberkulose, Lues u. dgl. Zähne geriffelt (rachitisch). Fuß beiderseits mit gutem Gewölbe. Rechter $F u B$ innen in der Gegend des Kahnbeins deutlich geschwollen (teigig, aber nicht fluktuierend), etwas warm und ganz leicht gerötet, ferner umschrieben stark druckempfindlich. An der Wade besteht leichte, aber deutliche Muskelatrophie.

$\begin{array}{lll}\text { Umfangsmaße: } & \text { rechts } & \text { links } \\ \text { Wade: } & 21 \mathrm{~cm} & 22 \mathrm{~cm} \\ \text { MittelfuB } & \text { I } 81 / 2, & 17^{1} / 2, .\end{array}$

An dem übrigen

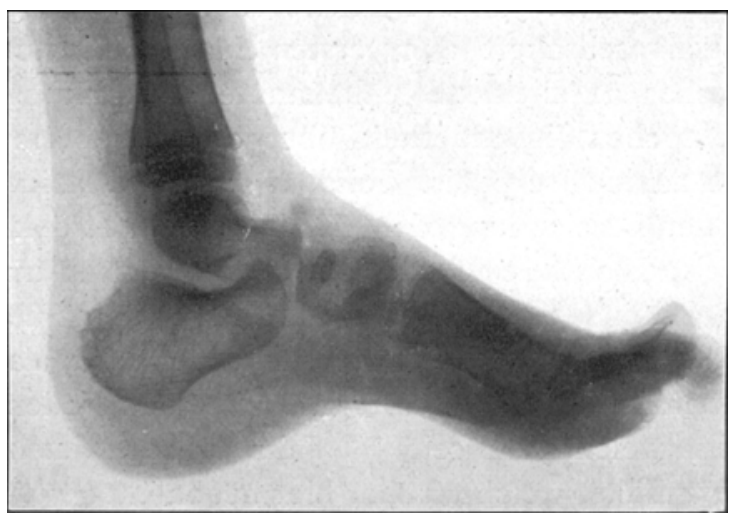

Fig. 1.

Fall I : Röntgenbild des gesunden Fußes.

Knochensystem, insonderheit an den Kniescheiben, kein krankhafter Befund. Kniescheibe beiderseits gut entwickelt; sie ist beiderseits je $4 \frac{1 / 2}{\mathrm{~cm}}$ lang und breit.

Röntgenbild ergibt: Kahnbeinknochenschatten rechts $(3 \times$ I I mm) gegen links $(6 \times 14 \mathrm{~mm})$ verschmälert und niedriger (auf ca. $1 / 2$ ), etwas unregelmäßig konturiert und stark verschattet unter Verlust der Knochenzeichnung. Kniescheibe beiderseits regelrecht. Epiphysenlinie am unteren Femurende beiderseits unregelmäßig.

Krankheitsverlauf: Zunächst wurde Bettruhe und feuchter Umschlag verordnet; daraufhin trat bereits nach Tagen deutliche Besserung auf, indem die Schwellung bedeutend zurückging und die Schmerzen fast verschwanden. Die weitere Behandlung bestand in Bädern, Massage und Schonung, sowie Verabreichung von Calcantabletten.

$\mathrm{Nachuntersuchung} \mathrm{nach} 1 / 4 \mathrm{~J}$ ahr: R. Fuß in der Kahnbeingegend noch etwas verdickt, aber nicht wärmer oder gerötet. Druckempfindlichkeit besteht noch ziemlich stark. Muskelatrophie deutlich; Umfangsmaße: Wade r. 22, 1. $23 \mathrm{~cm}$. Gang ist nicht deut- 
lich hinkend; Pat. tritt aber rcchts mehr auf den äußeren Fußrand; beiderseits geht er stark über die große Zehe.

Röntgenbild ähn. lich wie oben: $R$. $3 \times 15,1.7 \times 15 \mathrm{~mm}$, also auf der kranken Seite nunmehr etwas höher, sonst aber kaum verändert. Weiterbehandlung wie bisher und außerdem elastische Bindenwicklung.

$\mathrm{Nachunter}$. suchung nach 1/2 Jahr: In der Kahnbeingegend noch angedeutete Verdickung. Druckempfindlichkeit

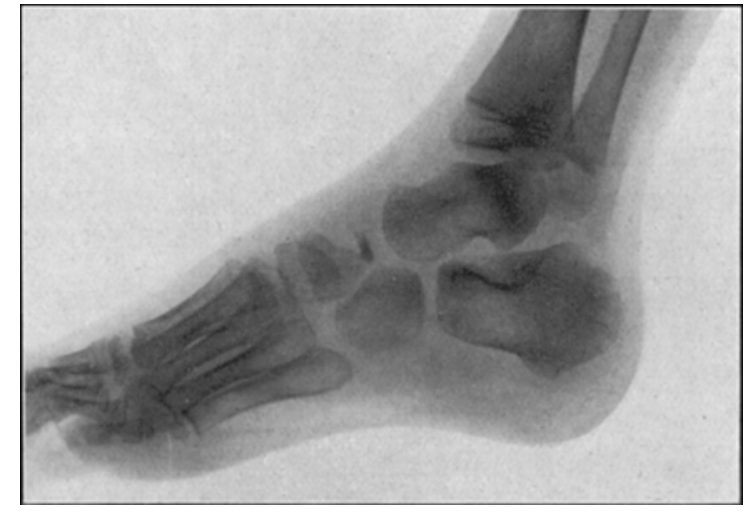

Fig. 2.

Fall I: Röntgenbild des kranken FuBes. kaum vorhanden. Muskelatrophie unverändert. Umfangsmaße: Wade r. $22^{1 / 2}, 1.23^{1 / 2} \mathrm{~cm}$. Gang nicht hinkend, auch nicht mehr auf dem äußeren Fußrand. Röntgenbild: Weiter erhöht, und zwar mehr wie 1 , aber Kahnbein r. $3 \times 17$ : 1. $7 \times 15 \mathrm{~mm}$, also aut der kranken Seite wie bisher verschmälert. auch mit unregelmä $B$ ger Zeichnung, speziell mit feiner Spaltandeutung und mit etwas Auf. lagerung geringeren Schattens nach dem Sprungbein zu.

Fall 2. H. K., $6 \mathrm{j}$. Knabe.

Vorgeschichte: Eltern gesund. Einziges Kind. Keine früheren Krankheiten, insonderheit keine Rachitis,

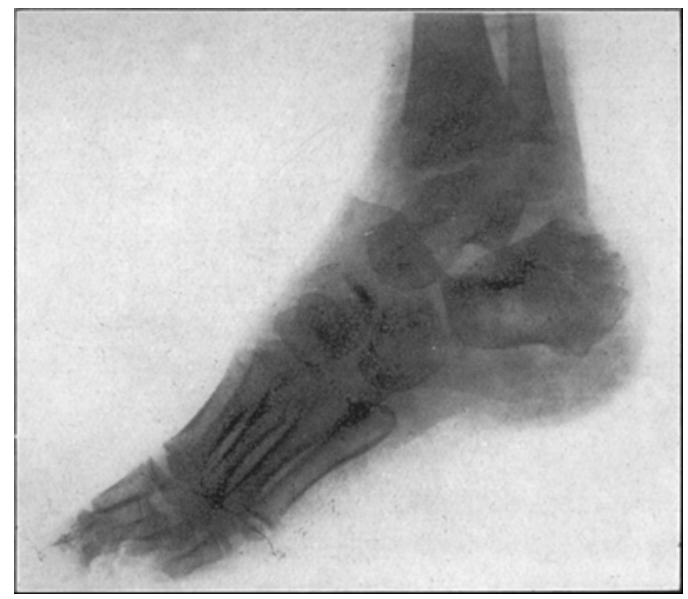

Fig. 3 .

Fall 1: Röntgenbild des kranken Fußes nach 1/4 Jahr.

Tuberkulose oder Lues. Zahnung und Laufen'ernen zur rechten Zeit. Frühjahr 1920 Masern; im Anschluß daran seit dem Aufstehen 
Schmerzen im r. Fuß innenseits und Auftreten nur mit dem äußeren Fußrand; ein Trauma wird in Abrede gestellt; jetzt bringt ihn der Vater, da die Beschwerden nicht nachlassen, in die hiesige Poliklinik.

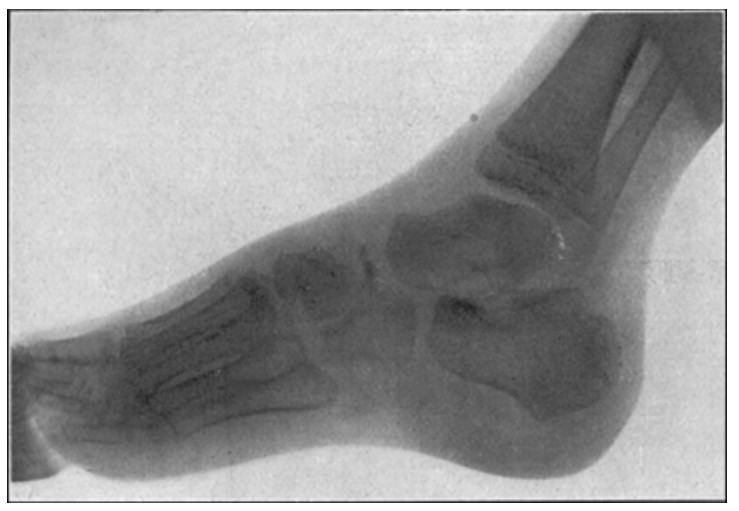

Fig. 4 .

Fall I: Röntgenbild des kranken Fußes nach 1/2 Jahr.

A uf nahme. befund: Allgemeinzustand o. B., insbesondere ohne Zeichen von Kör. perschwäche, Myxödem, Blutarmut, Rachitis, Tuberkulose, Lues u. dgl. Schneidezähne geriffelt (rachitisch). Fuß beiderseits mit mäßig gutem Gewölbe. L. Fuß innen in der Gegend des Kahnbeins ver dickt, mit ziemlich derber Weichtei!schwellung, aber nicht wärmer oder gerötet. Deutlich umschriebene Druckempfindlichkeit der Kahnbeingegend. An der Wade besteht

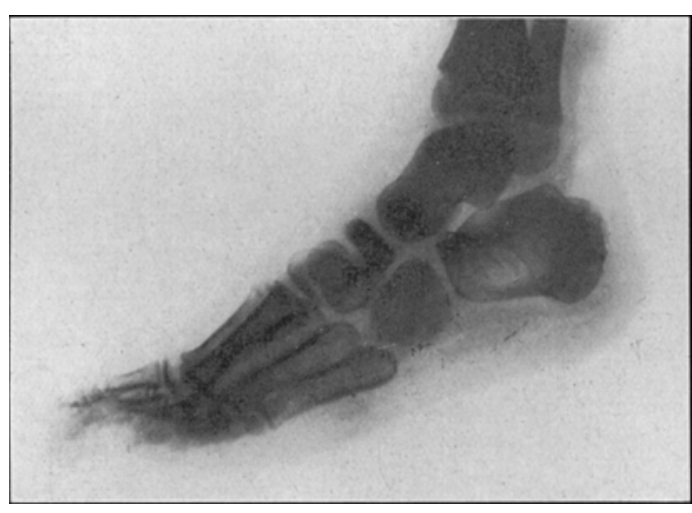

Fig. 5.

Fall 2: Röntgenbild des gesunden Fußes. mäßige, aber deutliche Muskeiatrophie. ITm. fangsmaße: Wade $r$. $2+, 1.23^{1 / 4} \mathrm{~cm}$. Schmer zen bestehen angeblich nicht. Wohl aber tritt Pat. mit dem äußeren Fußsrand auf; Hinken ist nicht dcutlich aus. gcprägt. An dem übrigen Knochensystem, insonderheit an den Kni.scheiben, kein krankhafter Befund; Knicscheibe ist bei. derscits gut entwickelt; sic ist beiderseits je $4 \mathrm{~cm}$ lang und breit; röntgenologisch regelrecht. Epiphysenlinien am untern Femurende unregelmäßig.

Röntgenbild ergibt: Kahnbeinknochenschatten lin'zs gegen rechts verschmälert und zugleich etwas erhöht $(7 \times 19$ gegen $9 \times 18 \mathrm{~mm})$, 
etwas unregelmäßig konturiert und stark verschattet unter Verlus.t der Knochenzeichnung.

Krankheitsverlauf: Zunächst wurde Schonung und feuchte Umschläge verordnet, nach 8 Tagen Schonung, Bäder, Massage, sowie Calcantabletten und elastische Wickelung.

Nachuntersuchung nach $1 / 4$ Jahr: L. Fuß in der Kahnbeingegend nicht mehr deutlich geschwollen, gerötet oder wärmer. Druckempfindlichkeit besteht noch. Muskelatrophie deutlich; Limfassungsmafe: Wade r. $24,1.23^{1 / 2} \mathrm{~cm}$. Gang ist zurzeit nicht hinkend, an-

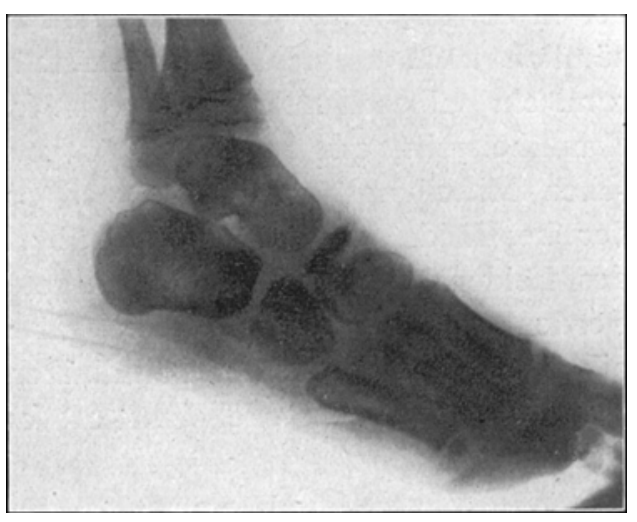

Fig. 6.

Fall 2: Röntgenbild des kranken Fußes. geblich überhaupt nur zeitweise. Pat. tritt nicht mehr deutlich nur mit dem äußeren Fußrand auf.

Röntgenbild: L. $8 \times 19$, r. $9 \times 18 \mathrm{~mm}$, also Kahnbein nunmehr auf der kranken Seite breiter, sowie Bälkchenzeichnung und Rindenausbildung andeutend.

Im Anschluß an die vorher beschriebenen Fälle soll eine kurze $\mathrm{Z}$ usammen stellung unserer derzeitigen Kenntnisse über das eigentümliche Krankheitsbild der Koehlerschen Affektion des Os naviculare pedis bei Kindern auf Grund der bisherigen Literatur gegeben werden.

Häufigkeit. Das Krankheitsbild scheint zwar

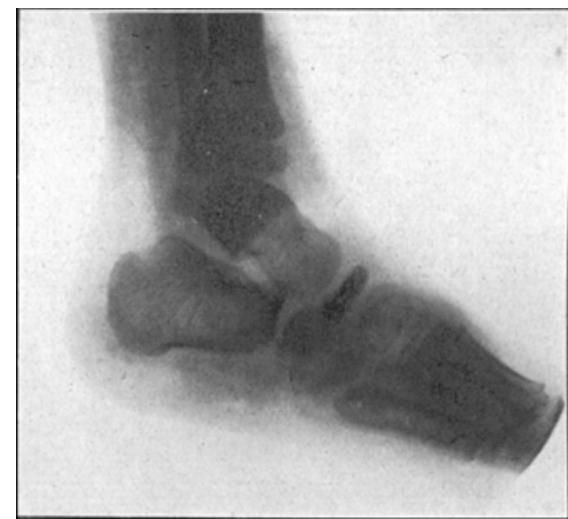

Fig. 7 .

Fall 2: Röntgenbild des kranken Fußes nach í Jahr. nicht häufig, aber auch nicht allzu selten zu sein. Wenigstens geht dies meines Erachtens aus der Tatsache hervor, da $B$ einzelne Beobachter bzw. Kliniken, welche ihr Augenmerk auf das Krankheits- 
bild richteten, in wenigen Jahren eine Reihe von Fällen zusammen stellen konnten, z. B. Beh n 5, Delorme 4, For sell 9, Koehle $\mathrm{r}$ 4, Schultze 7 und wir 4. Allerdings ist die Zahl der bisher in der Literatur veröffentlichten Fälle eine recht bescheidene, nämlich nach meiner Zusammenstellung bis 1920 nur 50, also rund ein halbes Hundert. Dabei ist freilich zu bedenken, da $B$ das Krankheitsbild erst seit der Röntgenära bzw. seit der Koehlerschen Mitteilung vom Jahre 1908 bekannt ist; auch dürfte bei der bescheidenen und operativ überhaupt fehlenden Bedeutung des Leidens nicht jeder Fall mitgeteilt werden, und schließlich werden wohl die meisten Fälle gar nicht in die Hand der Kli. niker, sondern in dic der Polikliniker und noch mehr in die der Praktiker gelangen, vielleicht oft auch immer noch verkannt werden.

Vorkommen: Was das Alter betrifft, so scheint das I.eiden nur vorzukommen bei Kindern zwischen 2 und Io Jahren, und zwar vorzugsweise bei solchen zwischen 5 und 9 Jahren, wie dies Koehler bereits seinerzeit angegeben hat, am häufig. sten wohl um das 5. bis 6. Jahr, während nur F orsell es auch schon früher fand. Es war betroffen von 27 genauer bekannten Fällen das Alter von

\begin{tabular}{|c|c|c|c|}
\hline 2 & Jahren & & nal \\
\hline 3 & , & 2 & ," \\
\hline 5 & , & 12 & ", \\
\hline $51 / 2$ & , & 2 & ,. \\
\hline 6 & , & t & , \\
\hline 7 & , & 2 & , \\
\hline $7 / 2$ & , & I & .. \\
\hline 8 & , & I & ,, \\
\hline $9^{1 / 9}$ & ,י & I & . \\
\hline 10 & , & 1 & , \\
\hline
\end{tabular}

Die Bevorzugung des genannten Alters hängt offenbar, worauf weiter unten eingegangen wird, mit der Knochenentwicklung des Kahnbeins zusammen.

Von den Geschlechtern war betroffen bei 36 genauer bekannten Fällen das männliche 25, das weibliche I I mal, also das männliche über doppelt so häufig als das weibliche; vielleicht hängt diese Tatsache mit der Finwirkung traumatischer Schäd- 
lichkeiten zusammen, welche bei Knaben ja häufiger und stärker zu sein pflegen als bei $M \ddot{a} \mathrm{~d} c h e n$.

Von den $\mathrm{Körperseiten} \mathrm{ist} \mathrm{die} \mathrm{rechte} \mathrm{vielleicht} \mathrm{etwas}$ häufiger befallen als die linke, ohne daß aber dieser Unterschied besonders auffallend wäre.

In einigen Fällen ist das Leiden doppelseitig, und zwar nach Koehlers im Jahre I 94 zusammengestellten 26 Fällen mindestens ro mal, also in über $1 / 3$ der Fälle; Schultze fand das Leiden dagegen in seinen 7 Fällen keinmal doppelseitig. Bisweilen ist der Röntgenbefund auf beiden Körperseiten zu erheben, aber nur auf einer Seite das klinische Krankheitsbild.

Symptome: Das klinische Krankheitsbild ist während des Höhestadiums ein typisches; es setzt sich in den allermeisten Fällen, wie auch in den meinigen, aus folgenden Symptomen zusammen:

1. Schmerz, und zwar spontan, bei Fußbewegungen und im Gehen; der Schmerz ist im ganzen ein mäßiger oder geringer, jedoch namentlich anfangs genügend stark, um das folgende Symptom hervorzurufen:

2. Hinken oder doch Schonung des in neren Fußrandes, also daher Auftreten in leichter Hohlfußstellung oder doch vorwiegend mit dem äußeren Fußrand.

3. Druckempfindlichkeit, und zwar am FuB innenund fußrückenseits in der Gegend des Kahnbeines; dieselbe wurde allerdings vermißt in den Fällen von $\mathrm{Haen}$ is $c h, \mathrm{Sch}$ äf fer und Schultze (3).

4. Weichteilschwellung, und zwar an der unter 3. genannten Stelle und von teigiger, vereinzelt fast fluktuierender Beschaffenheit, sowie erhöhte $\mathrm{Hauttemperatur}$ und leichte Rötung (letztere wurde einige Male notiert, fehlte aber auch öfters, kann übrigens auch bedingt werden durch therapeutische Maßnahmen, z. B. Jodtinkturpinselung).

5. Muskelatrophie am kranken Bein speziell Wade: über dieses Symptom finden sich bei den meisten Autoren leider keine Angaben; immerhin geht die Annahme wohl nicht fehl, daß dieses Symptom in mehr oder weniger hohem Grade regelmäßig vorhanden ist; ich fand in meinen beiden Fällen beidemal eine Umfangsverminderung der kranken Wade gegenüber der gesunden 
um I $\mathrm{cm}$ und Schäffer eine solche von $\mathrm{I} 1 / 2 \mathrm{~cm}$; aus dem Um. stande, daß in meinem Fall I bereits 2 Tage nach Beginn der Erkrankung die genannte Umfangsverminderung vorhanden war, wäre der Schluß zu ziehen, wenn derselbe bei der geringen Maßdifferenz erlaubt ist, daß eine Schonung und somit eine Erkrankung an dem betreffenden Bein schon länger bestanden hat, wenn auch nur in leichtem Grade.

Das Röntgenbild, wie es seinerzeit bereits von Koehle $\mathrm{r}$ und in Übereinstimmung mit ihm von den nachfolgenden Untersuchern beschrieben worden ist, ist ein ganz charakteristisches, welches dem Kundigen a uf einen Blick die Diagnose der Krankheit ermöglicht; die Charakteristika sind folgende:

Kahnbein ist $\mathrm{I}$. hinsichtlich der Grö $B$ e stark verkleinert, speziell verschmälert, und zwar dies durchschnittlich um ca. $2 \mathrm{~mm}$, ev. bis auf $1 / 2$ oder gar $1 / 4$ seiner Breite; was die Höhe angeht, so ist in einigen Fällen dieselbe auf der kranken Seite etwas erhöht gefunden worden, vgl. Grune, Stumm u. a., welche somit eine sagittale Abflachung und dazu senkrechte Erhöhung annehmen; man muß aber wohl wegen der Möglichkeit verschiedener Projektion bei kleinen Differenzen mit dem Urteil zurückhaltend sein; in unseren Fällen fehlte in dem einen eine Erhöhung, in dem anderen war sie vielleicht vorhanden, betrug aber höchstens I $\mathrm{mm}$; 2. hinsichtlich der Gestalt deformiert, bisweilen biskuitförmig und zugleich in den Umrissen unregelmäßig zackig; 3 . hinsichtlich der Struktur stark verdichtet, d.h. mit vermehrtem Schatten, und zwar dies bis auf das Zwei- oder gar Vierfache, und unregelmäßig, vorwiegend im Zentrum betroffen, und zugleich mehr oder weniger ohne Zeichnung der Architektur, spez. Bälkchen. Einige Autoren fanden außerdem eine Zerteilung des Knochenschattens: z. B. B a c h m a n n im I. Falle mehrere vom Hauptkern abgesprengte Gebilde in Form kleiner Schatten, welche von Einzelnen, wie von $S t u m m e$ als Frakturstücke gedeutet werden und welche später mit dem Hauptieile vereinigt waren, Grune Bruchlinien, Schultze im 6. Fall Kern zertrümmert und auch noch nach 9 Monaten 3 Frakturlinien und im 7. Falle zweiteiligen Kern, S t u m me dorsal kleines, schalenförmig abgehobenes und schräg gestelltes Corticalisstück, welches nach I Monat mit der Hauptmasse verschmolzen war. Im übrigen sei bezüglich der Einzel- 
heiten des Röntgenbildes auf die betreffenden Autoren verwiesen. Auf die De ut ung des Röntgenbefundes wird auch weiter unten bei Besprechung des Wesens der Krankheit eingegangen werden.

Diagnose: Differentialdiagnostisch kommt in Betracht: beginnender Plattfuß, Quetschung, Stauchung, Fraktur, Osteomyelitis und vor allem Tuberkulose; letztere ist in zahlreichen Fällen (vgl. Bles, Forsell, Koehler usw.) zunächst vom Kliniker angenommen worden und wird wohl anfänglich in $\mathrm{der}$ Regel vermutet. Das Röntgenbild klärt den Fall dann schnell auf; bei Kenntnis des Krankheitsbildes mit seinem charakteristischen Röntgenbefund ist eine Verwechslung mit anderen Leiden wohl nicht möglich. Aber auch schon $k \mathrm{lin}$ is $\mathrm{ch}$ dürfte die Diagnose durch den Kundigen wohl zu stellen sein, und zwar auf Grund der klinischen Symptome nebst Anamnese (Alter und Entstehung!)

Prognose, Komplikationen und Verlauf: Die Prognose muß nach den bisher beobachteten Fällen als eine durchaus gute bezeichnet werden. Komplikationen oder Folgezustände der Krankheit sind niemals verzeichnet, insonderheit nicht Osteomyelitis, Tuberkulose oder Tumor. Der Verla uf des Leidens, welcher am besten nach klinischen Symptomer: und nach Röntgenbild gesondert betrachtet wird, ist anscheinend stets ein zu völliger Heilung führender. Die klini$\mathrm{sch}$ er S y m p to m e verlieren sich meist schon in wenigen Wochen bis Monaten, und nach $1 / 4-3 / 4$ Jahr sind meist Beschwerden überhaupt nicht mehr vorhanden; die Angaben sind allerdings bei den einzelnen Autoren recht verschieden und im allgemeinen wenig bestimmt gehalten; so ist erwähnt bei:

Bachmann im r. Fall, ,nach 6 Wochen beschwerdefrei", im 2. Fall ,nach 8 Tagen keine Beschwerden", bei Schäffer, ,nach 8 Tagen auf, nach 9 Monaten geheilt", bei Schultze im 3. Fall, "nach 6 Wochen guter Gang", im 4. Fall, ,nach 6 Wochen Heilung“", im 5. Fall, "nach 4 Wochen guter Gang", im 7. Fall, „Gang mit Einlage bald gut", bei $\mathrm{mir}$, in 3 Tagen Beschwerden und nach $1 / 4 \mathrm{Jahr}$ auch klinische Symptome fast verschwunden“", bei Wohlauer ,in kurzer Zeit beschwerdefrei" usw.

Wie das $\mathrm{R}$ öntge n bild lehrt, findet anscheinend stets eine völlige Wiederherstellung statt, und zwar wird allmählich die Zeichnung deutlicher, der Schatten weniger dicht, die Form und 
Größe normal, so daß schließlich ein wohlgebildetes Knochenbild resultiert, dies spätestens nach $2-3$ Jahren, wie es seinerzeit Koehler bereits festgestellt hat (,normal nach 2-3 Jahren“) und zahlreiche andere Autoren bestätigen, z. B.:

B a chmann (im I. Fall, ,später normal“), Forsell (,zwei Fälle in $1 / 4^{-1 / 2} \mathrm{Jahr}$ normal"), Grashey, $\mathrm{H}$ a e nisch (,nach $1 / 2$ Jahr fast und nach $3 / 4$ Jahr ganz normal"), Schäffer (,nach 2 Jahren normal"), Schultze (,im I. Fall nach $1 / 2$ Jahr Zeichnung deutlich, nur wenig fleckig und etwas uneben konturiert; im 2. Fall nach $1^{1} / 4$ Jahr fast normal, nur im Kalkgehalt etwas verschieden von der anderen Seite; im 3. Fall nach I Jahre Kern noch flacher, unregelmäßig und fleckig, aber bereits besser ausgebildet; im 4. Fall nach $1 / 2$ Jahr Knochen zu 3/4 der normalen Höhe vergrößert, aber Ränder noch uneben und Aufhellungen im Inneren; im 6. Fall Kern so breit wie auf der anderen Seite und nicht mehr so fleckig wie früher"), Stumme ("nach $1 / 2$ Jahr Corticalis fast glatt, Spongiosa deutlicher, Aufhellung; nach $3 / 4 \mathrm{Jahr}$ Spongiosa deutlicher mit nur einzelnen dichteren Herden, Form und GröBe fast normal"), Wo h. l a u e r (,später normal“). Wie unsere beiden Fälle lehren, wird der Schatten auf der kranken Seite allmählich höher und langsam auch breiter; zugleich tritt eine Änderung der Zeichnung auf: Im ersten (schweren) Falle in Form herdweiser Aufhellung, im zweiten (leichteren) Fall in Form beginnender Bälkchenzeichnung und Rindenandeutung.

Therapie: Die Behandlung der Koehlerschen Krankheit ist gemäß ihrem im Verlauf zutage tretenden Charakter eine durchaus konservative. Operation ist bei sichergestellter Diagnose absolut kontraindiziert, wie dies bereits Koehler bemerkt. Meist genügt zunächst für einige Tage Bettruhe und feuchter Umschlag, später Schonung, Bindenwicklung, Bäder und Massage, sowie ev. Wärmeapplikationen, Stauen, Jodtinkturpinselung u. dgl. In schweren oder hartnäckigen Fällen wird ein Kontentiv-, z. B. Gipsverband, nötigenfalls in redressierter Stellung, ev. ein entlastender oder ein Schienenapparat empfohlen (Forsell, Ha enisch, Pfahler, Schultze, Stumme). Bei schlechtem Fußgewölbe kommt eine Plattfußeinlage in entsprechendem Stiefel in Betracht (vergleiche $\mathrm{Haenisch}, \mathrm{Schultze}$ ).

$\mathrm{Pathogenese:} \mathrm{Wie} \mathrm{bereits} \mathrm{Koehler} \mathrm{in} \mathrm{seiner} \mathrm{ersten} \mathrm{Ver-}$ öffentlichung bemerkt, handelt es sich bei unserem Leiden um ein wohl umrissenes Krankheitsbild im Sinne einer Krankheit sui generis. $\mathrm{Da} B$ es sich dabei nicht um Osteomyelitis, Tuberkulose, 
Lues oder dgl. handein kann, ist von $\mathrm{K}$ o e h le r damals ausführlich erläutert worden, worauf hiermit verwiesen sei. Auch die Deutung als Osteomalacie, welche $\mathrm{Kraft}$ in der Diskussion zu Koehlers Vortrag auf der 4. Tagung der Deutschen Röntgengesellschaft ausspricht, wird von Koehler sogleich abgewiesen und in der späteren Mitteilung widerlegt. Über das Wesen der Krankheit spricht sich Koehler in der I. Veröffentlichung nicht weiter aus.

In der Folge ist nun die Frage nach dem Wesen der Koehlerschen Krankheit Gegenstand lebhaften Meinungsaustausches geworden.

Ehe auf die umstrittene Frage nach dem Wesen eingegangen wird, sind eine Reihe von Tatsachen $z u$ erwähnen, welche einige Aufklärung $z \mathfrak{u}$ bringen geeignet erscheinen, insonderheit hinsichtlich der Ursache des Leidens.

Hereditäres Vorkommen ist bisher niemals beobachtet worden. DaB Krankheiten der Eltern und Voreltern eine Rolle spielen, ist nicht wahrscheinlich; zwar werden solche einige Male notiert, so z. B. von Koehler Gonorrhoe und von Bles, Koehler und Schultze je I mal Tuberkulose; jedoch stehen solchen, übrigens nicht viel besagenden positiven weitaus mehr negative Angaben gegenüber, nämlich die von Forsell (9), Haenisch (I), Schäffer (I), Schultze (6), wir (2), Woh la uer (3) und Wrede (I Fall).

Von voraufgegangenen Krankheiten werden erwähnt von $\mathrm{Behn}$ Enteritis, Bronchitis und Otitis media, von $\mathrm{Hae}$ $\mathrm{n}$ isch Masern, Skrofulose, Lymphome und Rachitis, von B a chmann Scharlach, von S chultze je einmal Scharlach und Mittelohrentzündung bzw. Diphtherie, von uns einmal Scharlach; hiernach kann von Zusammenhang des Leidens mit bestimmten anderen Krankheiten im allgemeinen nicht gesprochen werden. Wohl aber besteht die Möglichkeit, daß die Erkrankung nach anderen Krankheiten mit längerem Krankenlager sich dann entwickelt, wenn die Kinder wieder aufstehen und ungewohnterweise den Fuß wieder der Belastung aussetzen, wie dies in unserem 2. Fall wieder der Belastung aussetzen, anzunehmen ist.

Ferner erwähnt Koehler als auffallende Tatsache, daß mehrfach die Kinder in den ersten Monaten bis Jahren sehr 
schwächlich gewesen waren; diese Tatsache wird vermerkt von Bachmann (in I Fall). Behn, Bles, Forsell (in der Hälfte der Fälle), Haenisch, Koehler (in I Fall), Stumme; andererseits wird von einigen Autoren ausdrücklich betont, daß die Kinder kräftig waren.

$\mathrm{Rachitis}$ wurde beobachtet von $\mathrm{Behn}, \mathrm{Haenisch}$ und $\mathrm{St} \mathrm{umme}$. von den anderen Autoren dagegen niemals; auch in unseren Fällen bestanden keine Zeichen oder Reste florider Rachitis, wohl aber geriffelte Zähne; man darf hiernach wohl annehmen, daß die Krankheit jedenfalls nicht einfach als Folge der Rachitis angesprochen werden kann; immerhin besteht die Möglichkeit, daß Rachitis prädisponiert.

Was das $F u B g$ e wölbe angeht, so fand sich cin leichter Plattfuß 4 mal (Behn, Haenisch, Grune, Koehler), ein Hohlfub 2 mal (Bachmann, Koehler) und cin normaler Fuß anscheinend in den übrigen, mindestens in 12 näher bezeichneten Fällen (Bachmann. Koehler, Schultze, Stumme, wir).

Bemerkenswert ist der Fall von Koehler mit Myxödem bei einem 14jährigen Mädchen, welches die Skelettentwicklung eines Kindes von 5-10 Jahren hatte; es ist sehr verständlich, daß bei diesem Leiden, welches mit verzögertem Auftreten der Knochenkerne verbunden ist, die Entstehung der Krankheit begünstigt wird: der Fall ist bisher der einzige geblieben.

Ferner fand Kochler I mal die Patella beiderseits verkleinert, verunstaltet, verdichtet und ohne normale Architekturzeichnung. Auch Koritzinsky fand beide Kniescheiben aftiziert; Genateres uber den Befund dieses Autors ist aus dem Referat nicht zu ersehen. Von den anderen Autoren konnten verschiedene. welche darauf klinisch und röntgenologisch untersuchten, ein solches Vorkommnis in ihren Fällen nicht finden; speziell Schultze nicht in allen seinen 7 Fällen, Delorme nicht in seinen 4 Fällen (nach persönlicher Mitteilung) und auch wir nicht in unseren 2 Fällen.

In Grasheys Fall eines 8 jährigen Knaben war das $O_{s}$ naviculare manus beiderseits noch ohne Kern.

Was das Tra uma in der Vorgeschichte angeht, so finden sich über diese bei unsercm Leiden wichtige Frage bei den ein- 
zelnen Autoren folgende Angaben: Kein Trauma wird angenommen in ihren Fällen von Bachmann (2), Behn (5), Bles (I), Delorme (4), Forsell (9), Grashey (r), Koehler (4), Schäffer (1), Stumme (I), uns (2), Wohlauer (3), Wrede (1). Es sind dies 33 Fälle von i 1 Autoren; darunter sind höchstens 4 , in welchen möglicherweise cin leichtes Trauma eine Rolle spielte (nämlich im 2. Falle von $\mathrm{Bachmann: \text {vor }}$ 3 Wochen Sprung drei Treppenstufen herab mit Lremsen des Schwunges durch die rechte Ferse; in 2 Fällen von Forsell: Verstauchung und Steinstoß; im Fall von $S$ c hä f f e r : einige Tage zuvor Auffallen eines Backsteines). Ein Tra u ma wird dagegen angenommen in ihren Fällen von Grune is: Sprung $1 \mathrm{~m}$ tief die Küchentreppe hinunter auf den Großzzehenballen); Hae. nisch ( 1 ; Fall eines Gegenstandes auf den Fußrücken): Preiser 12; Treten durch Erwachsene auf die auf dem Erdboden stehenden FüBe); Schultze (7; vor 4 Monaten Sprung die Treppenstufe hinunter, vor 4 Wochen Spaziergang, vor 10 Wochen Sprung die Treppe hinunter, vor 4 Monaten fragliches Trauma, vor 3 Monaten $Z$ berfahrung des Fußes durch kleinen Handwagen, vor 4 Monaten Auffallen eines schweren Gegenstandes auf den Fußrücken, vor 4 Wochen Einklemmung in eine Tür); St u m me (I) Sprung rom Wagen); es sind dies I2 Fälle von 5 Autoren. Einige Male konnten wir über das Vorliegen eines Trauma keine Angabe: ausfindig machen, nämlich in den Fällen von L, o mon(I), Lorey (2) und P'fahler (1): es sind dies 4 Fälle von 3 Autoren. Zusammenfassend ist über die Frage des Trauma zu sagen: koehler fand 1914 kein Trauma angegeben mindestens in $2 / 3$ der Fälle, und zwar unter 26 bezeichneten in 16 , darunter höchstens cin geringes in 4; wir I 920 kein Trauma angegeben in fast $3 / 4$ der Fälle, und zwar unter 49 bezeichneten in 32 , darunter höchstens ein geringes in 4 , oder in anderer Fassung:

Fälle bezeichnete: $\left\{\begin{array}{c}\text { kein Trauma: } 34 \text { Fälle bei in Autoren / gleich ca. } 3 / 4 \\ \text { Trauma: } 12 \text {, }\end{array}\right.$ nicht bezeichnete:

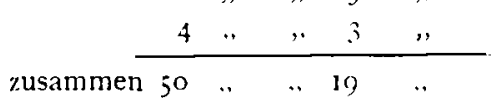

Dabei ist zu berücksichtigen, daß die Angabe „ob Trauma oder nicht" als eine relative aufzufassen ist; in den positiv verzeichneten Fällen handelt es sich zum Teil um geringe und um 
länger zurückliegende Traumen und in den negativ verzeichneten Fällen dürften solche auch nicht ausgeschlossen werden können, zumal es sich um Kinder handelt.

Nachdem vorgenannte Tatsachen aufgeführt sind, sei nunmehr aul die Frage nach dem Wesen der Krankheit, um welche sich ein Meinungsstreit unter den einzelnen Autoren erhoben hat, kurz eingegangen.

Eine verhältnismäBig kleine Anzahl von Autoren sieht in der Koehlerschen Krankheit die Folge einer Fraktur des $\mathrm{K}$ a $\mathrm{h} \mathbf{n}$ beines, und zwar einer Kompressionsfraktur. Beweisend erscheint ihnen dic in zahlreichen, speziell in ihren Fällen in der Vorgeschichte er. kennbare Entstehung durch ein Trauma einerseits und andererseits das Röntgenbild. Grune nimmt Kompressionsbruch an; er fanci das Kahnbein verlängert (4 $\mathrm{mm}$ ) und verschmälert i $2 \mathrm{~mm}$ ), im medialen Drittel von normaler Struktur, in den beiden übrigen Dritteln mit Verdichtung der Spongiosa und mit 2 Bruchlinien. Schultze vermutet ebenfalls Kompressionsbruch, allerdings je nach Intensität des Trauma in verschiedenen Formen: entweder Fraktur mit Kernkompression oder Kompressionsbruch oder Erweichungszyste; die Fraktur betrifft gerade und allein das Kahnbein um so eher, als das Kahnbein seinen Knochenkern am spätesten erhält und mit seiner Knochenentwicklung am spätesten fertig wird; ähnliche Bilder konnte er durch Traumen an der Leiche gewinnen; das von Koehler u. a. und auch von ihm in Fall 5 und 7 nach 6 bzw. 5 . Monaten gesehene Röntgenbild faßt er auf als in Heilung begriffene Stadien der Krankheit einige Zeit nach der Fraktur; für manche Fälle will er auch Knochenerweichung oder Ernährungsstörung oder Entzündung oder Wachstumsanomalie gelten lassen; für die von ihm beobachteten Fälle nimmt er Fraktur an und bleibt auch dabei in einer Erwiderung auf Koehler unter Hinweis auf Anamnese, Klinik und Röntgenbild. Stumme deutet das in seinem Fall gewonnene Röntgenbild ebenfalls als Kompressionsbruch wegen der sagittalen Abflachung und der dazu senkrechten Erhöhung (Maße sagittal r. 8, $1.6 \mathrm{~mm}$; vertikal r. 14,5, 1. $16 \mathrm{~mm}$; horizontal r. 15,5, 1. $16 \mathrm{~mm}$ ), Absprengung eines schalenförmig abgehobenen und nach i Monat mit der Hauptmasse verschmolzenen Corticalisstückchens, Verdichtung der zusammen- 
geschoben erscheinenden Spongiosabälkchen und Hinausrückens der 3 Keilbeinknochenkerne nach dem Sprungbein zu.

Inı Gegensatz zu diesen wenigen Autoren lehnt die Mehrzahl die Auffassung der Koehlerschen Krankheit als einer Fraktur ab. Koehler, welcher in seiner ersten Veröffentlichung auf die Deutung des Krankheitsbildes nicht eingegangen war, erkennt I 912 in der Diskussion zu Wohla uer eine traumatische Erkrankung nicht an unter Hinweis auf die mehrfach beobachtete Doppelseitigkeit und auf die in einem seiner Fälle beobachtete gleichzeitige Affektion beider Patellae, und bestreitet 1913 in einer Erwiderung auf $\mathrm{Schult}$ ze nochmals die Deutung seines Krankheitsbildes als Fraktur unter Hinweis auf die mehrfach (unter 26 Fällen mindestens $10 \mathrm{mal}$, also in $1 / 3$ der Zahl) beobachtete Doppelseitigkeit und auf das häufig (mindestens in $2 / 3$ der Fälle, nämlich unter 26 in 16 ) beobachtete Fehlen eines Trauma.

Grashey lehnt ebenfalls eine traumatische Entstehung ab. und zwar macht er unter anderm folgendes geltend: I. Die Nichtbeteiligung sonstiger Fußwurzelknochen spricht dagegen ; 2. Kernzertrümmerungen und Verdichtungen finden sich auch sonst, speziell an spät kommenden Knochenkernen, z. B. Fersenbeinkappe, Olekranon, Erbsenbein, Darmbeinkamm, Metatarsusbasis usw., ohne daß hicr ein Anhalt für traumatische Erkrankung anzunehmen sei. Aucl w wir fanden letztere Tatsache bestätigt, unter anderen an der Tuberositas tibiae bei einem Fall von Schlatterscher Krankheit. Schließlich sei auch auf die unten angeführte Angabe von $\mathrm{B}$ a de verwiesen, wonach das Kahnbein bisweilen aus cinem, bisweilen aber aus mehreren kernen verknöchert.

Auber $\mathrm{K}$ oehler und Grashey lehnen die Auffassung einer traumatischen Erkrankung bzw. Fraktur die meisten anderen Autoren ebenfalls ab, nämlich Ba ch man n, Behn, Delorme, Forsell, Pfahler, Wohlauer, Wredeu. a.

Wir möchten uns diesen Autoren anschließen; gegen Fraktur spricht u. E. außer den genannten noch der Umstand, daß das Röntgenbild, wenigstens in unseren Fällen, nicht das einer Fraktur ist, daß insbesondere Bruchstücke normal gebildeten Knochens, wie solcher auf der gesunden Seite erkennbar ist, ferner Bruchlinien, sekundäre Knochenatrophie usw. fehlen, umgekehrt in fort. dauernd aufgenommenen Nachuntersuchungen Bilder erkennbar 
sind, welche als Verknöcherungsstörung und -wiederherstellung zu deuten sind (s. o.).

Auch ergibt die klinische Intersuchung nicht cin Zuwenig an der Kahnbeingegend, was bei einem Kompressionsbruch in Form der beigezogenen Röntgenbilder erwartet werden mülßte, sondern (ähnlich wie bei der Schlatterschen Krankheit an der Tuberositas tibiae) ein $\measuredangle$ uvi el, was im Verein mit den Röntgenbildern an eine Wucherung des präossalen Knorpels denken läß (vgl. I)elorme).

Somil darf die Auffassung der Koehlerschen Krankheit als einer einfachen Fraktur wohl abgelehnt werden, wenigstens für die meisten Fälle. In einigen wenigen Fällen mag immerhin eine Fraktur vorliegen. In der Regel handelt es sich wohl nach der Meinung der meisten Autoren vielmchr um eine Knochenentwicklungsstörung, also Wachstumsanomalie. Dabei mag das Trauma eine gewisse auslösende oder begünstigende Rolle spielen, zumal Traumen im kindlichen Alter ja häufig sind, auch in einer groben Zahl der beschriebenen Fälle Traumen, spe\%iell länger voraufgegangene und leichtere (z. B. Sprung von Wagen oder Treppe. Auffallen von Lasten, Überfahren, Einklemmen, Auftreten auf den Fußrücken, langer Spaziergang u. dgl.) erwähnt werden und solche Traumen bei dem späteren Aufteten und lintwickeln des Knochenkenes im Kahnbein gerade dieses wohl bevorzugen können.

(ranz besonders erscheint bemerkenswert, dal3 das Kahnbein in der Stoßachse des Fußes beim Sprung auf die Fußspitze liegt derarı, daß dabei der Stoß von dem Metatarsalbein über das Kahnbein fortgeleitet wird. Es ist dies derselbe Mechanismus, welcher wohl ofters auch zur Entstehung des Kahnbeinbruches führt (igl. Günther, Zur Pathologie des Os naviculare pedis, Zeitschr. f. Röntgenkunde 1911, Bd.13), und es ist ja gerade der sprung auf die Zehenballen, welcher bei Kindern, z. B. beim Treppenherabspringen sicher häufig und energisch stattfindet, wie dies ja auch aus den anamnestischen Angaben zahlreicher Fälle herausgelesen werden kann.

Was Auftreten und Entwickeln des Knochenkerns im Kahn. bein angeht, so seien einige entwicklungsgeschichtliche Daten beigezogen: Nach Langer-Toldt erscheint der Knochenkern im 
Kahnbein des Fußes im 5. Jahr (vgl. Delorme). Nach Sick tritt im Kahnbein der Knochenkern am spätesten von allen Fuß. wurzelknochen auf, meist im 3. Jahr, aber in recht wechselndem Alter, z. B. war er bei 2 Jahren bereits normal und bei 4 Jahren noch fehlend. B a de bemerkt:

„Das Kahnbein ist das letzte von den Tarsalknochen, welcher auf dem Röntgenbild erkennbar wird. Seine Ossifikation scheint auch zeitlich keine ganz gleichmäßige zu sein, wenigstens geben die Embryologen verschiedene Daten seines Auftretens an: Koellicker das I., Gege n ba uer das 3.-5. Jahr. Auch ergeben sich aus den Bildern qualitative Verschiedenheiten seiner Ossifikation; bisweilen ist es aus 1 , bisweilen aber aus 2 Kernen entstehend. Bei einem älteren ( $5 \mathrm{j}$.) Mädchen war es noch recht klein."

Die Rolle des Trauma als eines zwar nicht ursächlichen, aber möglicherweise die Krankheit auslösenden und begünstigenden Momentes ist oben gewürdigt worden; namentlich $\mathrm{Hae} \mathrm{n}$ is $\mathrm{ch}$ und Preiser räumen dem Trauma einen wesentlichen Einfluß ein im Sinne einer Störung der Knochenentwicklung; Preiser nimmt eine traumatische Ernährungsstörung eines intrakapsulär gelagerten kurzen Knochenkernes an, stellt sie in Parallele zu gewissen traumatischen Ernährungsstörungen der Handwurzel, speziell des Kahn- und Mondbeines und schlägt als Krankheitsbezeichnung „traumatische Ernährungsstörungen der Hand- und Fußwurzelknochen" vor; auch mag an dieser Stelle unter anderen Kien. b ö cks Beschreibung einer cigentümlichen traumatischen Erkrankung des Os lunatum erwähnt werden, wobei von einzelnen Chirurgen Kompressionsfraktur angenommen wird, wobei aber die Diagnose Fraktur nicht sicher erscheint, sondern ebenso wahrscheinlich eine Ernährungsstörung des Knochens und wobei ähnliche Röntgenbilder wie bei unserer Erkrankung vorliegen.

Welcher Art letzten Endes das Wesen der Koehlerschen Krankheit ist, ist heute noch nicht geklärt; über Vermutungen ist man bisher nicht hinausgekommen. Als wahrscheinlich kann wohl gesagt werden, daß eine Knochenentwicklungs störung vorliegt und daß möglicherweise Traumen, und zwar mäBige, diese Knochenentwicklungsstörung auslösen oder begünstigen. In Übereinstimmung dieser Annahme steht die Tatsache, daß Knochenentwicklungsstörungen bzw. Verzögerung der Epiphysenverknöcherung an verschiedenen Körperstellen, z. B. an der 
Tuberositas tibiae (Schlattersche Krankheit) ganz ähnliche klinische Erscheinungen machen; es liegt dabei nahe, an Schädigung der Knorpelmarkkanäle bzw. deren Gefäße zu denken, welche nach M. B. Schmidt für den normalen Ablauf der Ossifikation und nach $S \mathrm{chmorl}$ auch für den Eintritt der präparatorischen Knorpelverkalkung von Bedeutung sind. Im übrigen liegen folgende Meinungsäußerungen vor: Delorme vermutet Vermehrung des präossalen Knorpels im Beginn einer knöchernen Umwandlung, also eine Krankheit nicht des Knochenkernes, sondern seines Muttergewebes, und als Ursache davon eine Ernährungsstörung, ausgelöst durch Trauma, und zwar durch fortgesetztes auf das infolge seiner Einlagerung in den medialen Fußstrahl gefährdete Kahnbein, z. B. bei Zehenstand oder Sprung auf Vorderfuß, sowie Zug und Druck der Muskeln und Bänder; für diese Auffassung spreche auch die baldige Wiederherstellung des Knochenbefundes und die Tatsache, da $\beta$ bei einem gjährigen Kind wohl die klinischen Symptome vorhanden waren, nicht aber das typische Röntgenbild, wohl zu erklären durch das in diesem Falle spät erfolgte Einsetzen der Krankheit bei bereits vollendeter Knochenumwandlung des Kahnbeines.

Preiser, wie schon oben vermerkt wurde, stellt die Koehlersche Krankheit am Kahnbein des Fußes in Parallele zu gewissen traumatischen Ernährungsstörungen der Handwurzelknochen, speziell des Kahn- und Mondbeines. W r e d e setzt sie in Analogie zu der Schlatterschen und zu der Perthesschen Krankheit. Auch Koeh ler weist hin auf die Perthessche Krankheit. Mir will die Schlattersche Krankheit analog erscheinen, namentlich im klinischen Bilde. Ob etwa Spätrachitis zugrunde liegt, wie Fromme annimmt, ist nach den bisherigen Beobachtungen auch für die Koehlersche Krankheit möglich, aber nicht bewiesen.

B a chmann, welcher keine Fraktur, sondern Entwicklungsstörung annimmt, weist hin auf den pathologischen Befund an den Epiphysenlinien speziell in seinem ersten Fall, wo die untere Tibiaepiphyse verbreitert und unregelmäßig erschien.

Auch unsere beiden Fälle lassen Beziehungen zur Rachitis und zum Habitus asthenicus vermuten, wie solches bei der Schlatterschen Krankheit nach fremden und eigenen Beobachtungen ebenfalls angenommen werden darf; auf Rachitis verweisen aller- 
dings nur die geriffelten Schneidezähne; Röntgenbilder an den unteren Femurenden ergeben unregelmäßige Epiphysenfuge. Die Rolle, welche die Rachitis spielt, kann freilich so aufgefaßt werden, daß sie eine Prädisposition für die Entstehung der Koehlerschen Krankheit bildet. In dem einen Fall Koe hlers findet die Knochenentwicklungsstörung eine wahrscheinliche Prädisposition in dem gleichzeitig bestehenden Myxödem, zumal das 14jährige Mädchen die Skelettentwicklung eines 5-rojährigen Kindes bot; dieser $F$ all ist aber bisher vereinzelt und läßt keinen unmittelbaren Schluß zu für die übrigen Fälle, in denen Anzeichen für Myxödem fehlten; immerhin ist der Fall bemerkenswert.

\section{$\mathrm{Z}$ us a mmenf assung.}

Mitgeteilt werden 2 Fälle von Koehlerscher Krankheit bei 6jährigen Knaben mit typischen klinischen Symptomen und mit typischem Röntgenbild.

Im Anschluß an diese selbst beobachteten Fälle wird eine kurze Darstellung des Krankheitsbildes der Koehlerschen Krankheit an Hand der Literatur gegeben:

$\mathrm{Hä} \mathrm{ufigkeit} \mathrm{der} \mathrm{Krankheit} \mathrm{scheint} \mathrm{nicht} \mathrm{allzu} \mathrm{selten} \mathrm{zu} \mathrm{sein;}$ aus der Literatur konnten einschließlich der zwei selbst beobachteten im ganzen 50 Fälle gesammelt werden.

Was das Vorkommen angeht, so betrifft das Leiden Kinder $z$ wischen 2 und 10 , meist zwischen 5 und 9 Jahren; das männliche Geschlecht ist doppelt so häufig befallen wie das weibliche; eine Körperseite erscheint nicht auffallend bevorzugt; mehrfach tritt das Leiden doppelseitig auf.

Klinische Symptome sind meist: Schmerz, Hinken, Druckempfindlichkeit, Weichteilschwellung, ev. auch erhöhte Hauttemperatur und leichte Röte und wahrscheinlich auch Muskelatrophie.

Röntgenbild ist charakteristisch, indem das Kahnbein verschmälert, unregelmäßig gestaltet und verdichtet erscheint.

Differentialdiagnostis $\mathrm{ch}$ ist Tuberkulose häufig in Betracht gekommen; die richtige Diagnose ist auf Grund der klinischen Symptome und Anamnese wohl möglich und auf Grund des Röntgenbildes sicher.

Prognose ist gut; Komplikationen sind bisher nicht be- 
obachtet; die klinischen Symptome verlieren sich in Tagen bis Wochen und im Röntgenbild ist spätestens nach 2-3 Jahren völlige Wiederherstellung bisher in den nachuntersuchten Fällen immer gefunden worden.

Therapie ist konservativ.

$\mathrm{Pathogenese} \mathrm{ist} \mathrm{noch} \mathrm{ungeklärt.} \mathrm{Wahrscheinlich} \mathrm{handelt}$ es sich um Knochenentwicklungsstörung. Traumen, speziell mäßige spielen möglicherweise bzw. wahrscheinlicherweise eine auslösende Rolle. Fraktur ist in einzelnen Fällen vielleicht nicht ganz auszuschließen, aber für die Mehrzahl der Fälle abzulehnen.

\section{Literaturverzeichnis.}

I. B a chman n, Disk. zu Koehler. Verh. d. Deutsch. Röntgengesellsch. I9I4, Bd. 10, S. 202.

2. B a de, Die Ossifikation des menschlichen Fußskeletts nach Röntgenogrammen. Fortschr. a. d. Geb. d. Röntgenstrahlen I899/1900, Bd. 3. S. 134 .

3. Behn, Isolierte Erkrankung des Naviculare pedis bei Kindern als Zeichen einer Wachstumsstörung. Fortschr. a. d. Geb. d. Röntgenstrahlen $1909 / 10$, Bd. I4, S. 262.

4. --, Disk. zu Koehler. Verh. d. Deutsch. Röntgengesellsch. 1914, Bd. I0, S. 202.

5. Bles, Die Koehlersche Knochenerkrankung. Münchner med. Wochenschr. 1913, Nr. 35, S. 1940.

6. Delorme, Disk. zu Koehler. Verh. d. Deutsch. Röntgengesellsch. I9i4, Bd. 10, S. 202.

7. Dob is ch, Zur Atiologie dır Koehlerschen Knochenerkrankung. Mün. chener med. Wochenschr. Igc8, Nr. 44, S. 228j.

8. Fors el1, Disk. zu Wohlauer. Verh. d. Deutsch. Röntgengesellsch. Igr2, Bd. 8 , S. 37 .

9. Fromme, Neuere Untersuchungen über Spätrhachitis. Verein nordostdeutscher Chirurgen 5. XII. I919, ref. Zentralbl. f. Chir. 1920, Nr. 20, S. 235.

I0. Gra sh e y, Disk. zu Koehler. Verh. d. Deutsch. Röntgengesellsch. 19i4, Bd. 10, S. 202.

I1. -, in Rieder-Rosenthal, Lehrbuch der Röntgenkunde, Leipzig 1913, S. $\log$.

12. Grune, Ein Fall von isolierter Kahnbeinfraktur des Fußes. Deutsche Zeitschr. f. Chir. 1913, Bd. I21, S. 195. 
13. Haenisch, Uber eine häufige, bisher anscheinend unbekannte Erkrankung einzelner kindlicher Knochen. Münchner med. Wochenschr. 1908, Nr. 46, S. 2377.

14. - Disk. zu Wohlauer. Verh. d. Deutsch. Röntgengesellsch. 19ı2, Bd. 8, S. 39 .

I5. K i e nbö ck, Traumatische Ernährungsstörung der kurzen Hand- und Fußwurzelknochen. Fortschr. a. d. Geb. d. Röntgenstrahien 1918/19, Bd. 16 und Zentralbl. f. Chir. 1911, Nr. 12, S. 45I.

16. Koehler, Über eine häufige, bisher anscheinend unbekannte Erkrankung einzelner kindlicher Knochen. Verh. d. Deu'sch. Röntgengesellsch. 1908, Bd.4, S. 110 und Münchner med. Wochenschr. 1908, Nr. 37, S. 1923 .

17. -. Disk. zu Wohlauer. Verh. d. Deutsch. Röntgengesellsch. 1912, Bd. 8, S. 40.

18. -. Das Koehlersche Knochenbild des Os naviculare pedis bei Kindern -- keine Frakturl Arch. f. klin. Chir. 1913, Bd. 101, S. 560.

19. - Zur Pathologie des Os naviculare pedis der Kinder. Verl. d. Deutsch. Röntgengesellsch. 1914, Bd. 10, S. 200.

2o. Koritzinsky, Koehlersche Krankheit. Norsk. Mag. f. Laegevidensk. 80, ref. Deutsche med. Wochenschr. I920, Nr. 5, S. 138.

2r. K raft, Disk. zu Koehler. Verh. d. Deutsch. Röntgengesellsch. 1908, Bd. 4, S. Iro.

22. L o m o n, Atrophie du scaphoide et pied plat. Bull. et mém. de la soc. de rad. méd. de Paris Igl I, zit. Koehler.

23. Lorey, 2 Fälle von Koehlerscher Krankheit. Ärztl. Verein Hamburg, 8. III. 1919, ref. Münchner med. Wochenschr. 1919, Nr. 15, S. 428.

24. Pfahler, Erkrankung des Naviculare. Surg., gyn. and obster. I9i3, ref. Fortschr. a. d. Geb. d. Röntgenstrahlen 1913/14, Bd. 23, S. 108.

25. Pre is er, Zur Frage der typischen traumatischen Ernährungss örungen der kurzen Hand- und Fußwurzelknochen. Fortschr. a. d. Geb. d. Röntgenstrahlen 1911, Bd. 17, S. 360 .

26. -, Disk. zu Wohlauer. Verh. d. Deutsch. Röntgengesellsch. 1912, Bd. 8, S. 38.

27. Schäffer, Die Koehlersche Knochenerkrankung. Münchner med. Wochenschr. 1910, Nr. 29, S. I548.

28. Schultze, Das Alb. Koehlersche Knochenb:ld des Os naviculare pedis bei Kindern - eine Frakturl Arch. f. klin. Chir. I912, Bd. I00, S. 431. 29. -, dgl. 1912, Bd. 101, S. 564 .

30. Sick, Die Entwicklung der Knochen der unteren Extremität von der Geburt bis zum vollendeten Wachstum. Arch. u. Atlas der norm. u. path. Anatomie in typischen Röntgenbildorn. Fortschr. a. d. Ge'J. d. Röntgenstrahlen 1902, Erg.-Bd. 9. 
31. Stieda, Disk. zu Koehler. Verh. d. Röntgengesellsch. I908, Bd. 4, S. 110.

32. Stumme, Kompressionsfraktur des Knochenkerns des Os naviculare pedis. Ein Beitrag zur sog. Koehlerschen Knochenerkrankung. Fortschr. a. d. Geb. d. Röntgenstrahlen I9ro/ı I, Bd. I6, S. 342.

33. Wohlauer, Uber die Koehlersche Erkrankung des Os naviculare pedis. Verh. d. Deutsch. Röntgengesellsch. 1912, Bd. 8, S. 36 und Ir. Kongreß d. Deutsch. Gesellsch. f. orthopäd. Chir. I9I2.

34. Wrede, Ein Fall von Koehlerscher Krankheit. Naturwiss.-med. Ges. Jena, I. II. I912, ref. Münchner med. Wochenschr. 1912, Nr. 12, S. 666.

Nachtrag bei der Korrektur: Nachuntersuchung nach weiterem $1 / 4 \mathrm{Jahr}$, also im 1. Fall nach $3 / 4$ und im 2, nach $1 / 2$ Jahr ergab keine wesentliche Anderung des Befundes, und $z$ war weder des klinischen noch des röntgenologischen.

An we it e re n B e it $r$ äge n fanden sich in der neuesten Literatur folgende:

35. Behm, Beitrag zur Kochlerschen Erkrankung des Os navic. pedis boi Kindern. Fortschr. a. d. Geb. d. Röntgenstrahlen 1921, Bd. 27, S. 628: I Fall bei 63/4 j. Knaben; Operation: Kahnbcinexstirpation; darauf schnelle Heilung. Kahnbein erwies sich äuBerlich nicht verändert, spez. nicht abgeplattet. Knorpel im Querschnitt (statt rötlich) gclblich, Knochenkern trocken und bröcklig. Histologisch fand sich Knorpel vom normalen Bau des hyalinen Knorpels; Knochen. mark normal, aber darin verstreut strukturlose und kaum gefärbte Stellen (nekrotische Spongiosa bälkchen?); nirgends Osteoklasten (aseptische Knocheinnekrose?). Röntgenbild sonstiger Knochen ergab Knochenkernentwicklung entsprechend 4 j. (statt 63/.j.) Knaben. Pathologisch handeit es sich wohl um Knochenentwicklungsstörung im Sinne von Verzögerung der enchondralen Ossifikation; Trauma wird als Ursache abgelelınt, höchstens als ein die Knochengefäße schädigenres Yoment ancrkannt.

36. M e u l e $\mathrm{gracht}$, Dit Kochlersche Krarıkheit im Os navic. b i Kindrin. Hospitalstidende Bd. 58: 5 Fälle; Trauma in allen Fällen als Ursache unwahrscheinlich, höchstens in 2 Fällen von Bedeutung im Sime auslösenden Moments.

37. Mouchet u. Roederer, La scaphoidite tarsicnne des jeunes enfants. Rev. d'orthop. 1920 ref. Zentralbl. f. Chir. 1921, Nr. 15, S. 551: Vorschlag der Bezeichnung ,Scaphoiditis tarsalis bei kleinen Kindern"; traumatische Entstehung erscheint nicht wahrscheinlich; vielleicht handelt es sich um eine pathologische Fraktur, welche durch Dystrophie des Kahnbeins vorbereitet ist; 36 Fälle aus der Literatur, davon 4 eigene.

38. Weil, Uber die Bezichung der Osteochondritis def. coxae juv. und der Alban Koehlerschen Krankheit. Beitr. z. klin. Chir. 192r, Bd. 122, S. 4t8, Zentralbl. f. Chir. 1921, Nr. I5, S. 517 u. Nr. 16, S. 576: I Fall bei 7 j. Knaben, welcher vor 8 Tagen über Turnschnur gesprungen war; Operation: Kahnbeinexstirpation. Histologisch fanden sich im Präparat keine Zeichen von Entzündung oder Trauma, sondern gleichmäBige Knorpelmasse mit schr kleinem Kern von Spongiosa in unregelmäBiger Anordnung und von besonderer Dichtigkeit (,als wenn der abnorm kleine Knochenkern die ganze Kalkmasse des sonst viel größezen normalen Knochenkerns in sich gesammelt hätte"). Vermutet werden Bcziehungen zur Schlatterschen Krankheit und zur Mondbeinmalacir, sowie vor allem zur Perthesschen Krankheit, was für letztere ausführlich begründet wird. , Abgelehnt wird Entzündung (Scaphoiditis der Franzosen) und Trauma; pathogenetisch handelt es sich wahrscheinlich um fötale Störung der Verknöcherung teils infolge kongenitaler Ursache (intrauteriner Druck?), teils infolge postfötalcr Ursache (z. B. Hypothyreoidismus); vorgeschlagen wird Bezeichnung als dysplastische Malacie. 